\title{
A model-reference impedance control of robot manipulators using an adaptive fuzzy uncertainty estimator
}

\author{
Gholamreza Nazmara $^{1}$, Mohammad Mehdi Fateh ${ }^{1}$, Seyed Mohammad Ahmadi ${ }^{*}$ \\ ${ }^{1}$ Department of Electrical and robotic engineering, Shahrood University of Technology, Shahrood, Iran, \\ E-mail: rezanazmara89@gmail.com \\ ${ }^{1}$ Department of Electrical and robotic engineering, Shahrood University of Technology, Shahrood, Iran, \\ E-mail:mmfateh@shahroodut.ac.ir \\ ${ }^{1}$ Department of Electrical and robotic engineering, Shahrood University of Technology, Shahrood, Iran, \\ E-mail: s.m.ahmadi1365@gmail.com
}

Received 12 January 2017

Accepted 18 April 2018

\begin{abstract}
This paper aims at developing a voltage -based impedance model-reference controller using fuzzy uncertainty estimator for the robust control of electrically driven robot manipulators. The proposed control scheme not only utilizes a desired impedance as a reference model, but also provides the integrated control of position and force in the closed loop system. The robotic system receives the output of model-reference as a desired trajectory and thus the aim of the controller is to reduce the difference between the task-space desired trajectory and the desired impedance model. Furthermore, two control terms namely a robustifying term and a fuzzy uncertainty estimator are added in the structure of control design in order to improve the performance of the control system as well as to tackle uncertainty including un-modelled dynamics and external disturbances. Using stability analysis, we derive the adaptive mechanisms and also prove the boundedness of all system states. Finally, simulation results are included to verify the proposed éntrol method.
\end{abstract}

Keywords model-reference, impedance control, electrically driven robot manipulators, fuzzy uncertainty estimator.

\section{Introduction}

Many researchers have focused attention on designing force control schemes for robot manipulators in the past decades. As an end-effector of robot manipulators interacts with an environment, the existing contact force must be regulated to protect the end-effector and environment against serious damages. To solve this problem, the hybrid position/force control approaches [12] have been proposed to guarantee a smooth transition from position control to force control. However, the formulation of hybrid position/force control cannot be easily achieved. Also, many of these control schemes require the exact kinematics and dynamics of robotic system; hence, an adaptive Jacobian position/force control [3] has been developed which does not require all the knowledge of the robot manipulator.

In order to provide a unified solution of the position and force control, the impedance control schemes [4-6] have been developed for robot manipulators. An impedance model is designed to describe the dynamic behavior of a robotic system when interacting with the environment. It is worth noting that impedance control methods are strongly affected by the uncertainty associated with the nonlinear dynamics of highly coupled robotic systems, unknown environments and external

${ }^{*}$ Corresponding author. 
disturbances [7]. Therefore, the performance of control system is dependent on how well the uncertainty can be compensated [8]. Adaptive impedance controllers [9], robust impedance controller [10], learning impedance control [11] and force tracking impedance control [12] have been developed to tackle uncertainty. It should be emphasized that the majority of impedance controllers are based on torque control strategy such that the proposed control laws require the dynamics of robot manipulator and also the role of robot's motors is excluded from the control problem.

To consider the whole robotic system including robot manipulator and motors, a regressor-based variablestructure controller [13], the min-max scheme [14] and a generalized impedance controller [15] have been proposed. By using the voltage control strategy [16-17], the impedance control of electrically driven robots has been proposed [18]. The main idea behind the voltage control strategy comes from the fact that the manipulator dynamics can be taken into account indirectly as measuring the motor currents.

An adaptive impedance control of human-robot cooperation [19] has been developed. In this control method, linear quadratic regulation has been formulated to minimize tracking errors and to obtain an optimal impedance mode of human. An adaptive neural impedance controller [20] for robot manipulators has been proposed by considering uncertainty and input saturation. An adaptive control method [21] has been designed for tracking purpose of uncertain robotics in order to compensate parametric uncertainties, unmodelled dynamics and external force disturbances without a priori knowledge about upper bound of uncertainty. An adaptive fuzzy neural network force approach [22], robust neural network [23] and robust adaptive neuro-fuzzy controller [24] for hybrid position/force control of robot manipulators have been developed, which interacts with unknown environment. A PID-fuzzy controller was developed to deal with the nonlinear contact force control problem [25].

This paper presents a voltage-based model-reference impedance control approach using a fuzzy uncertainty estimator for the electrically driven robot manipulators. In the proposed control scheme, a desired impedance is used as a model-reference in such a way that this modelreference could provide a reference signal for a robot in both tracking and contact situations. As the interaction between an end-effector and an environment occurs, the contact force is exerted to the model-reference and the robotic system receives the output of model-reference as a desired trajectory and thus the aim of the controller is to reduce the difference between the task-space desired trajectory and the desired impedance model. In order to tackle difficulties in the analysis of the motion equation in task-space, a specific transformation is used and a pertinent controller is designed in the joint-space. Furthermore, two control terms namely, a robustifying term and a fuzzy uncertainty estimator are employed in the proposed controller: (i) to improve the performance of control system; (ii) to compensate un-modelled dynamics and external disturbances.

In the remainder of this paper, the robotic problem formulation is discussed in Section 2. In section 3, fuzzy uncertainty estimation is introduced. Section 4 develops the impedance design. The proposed robust control approach and the stability analysis of the control system are developed in section 5. Section 6 illustrates the corresponding simulations to validate the theoretical results. Finally, Section 7 concludes the paper.

\section{Problem Formulation}

The dynamics of a robotic system which is in contact with a frictionless rigid environment consist of $n$ links interconnected at $n$ joints into an open kinematic chain of rigid links and the geared permanent magnet dc motors are described as [26]

$$
\begin{gathered}
\boldsymbol{D}(\boldsymbol{q}) \ddot{\boldsymbol{q}}+\boldsymbol{C}(\boldsymbol{q}, \dot{\boldsymbol{q}}) \dot{\boldsymbol{q}}+\boldsymbol{G}(\boldsymbol{q})+\boldsymbol{J}^{T} \boldsymbol{F}_{e}=\boldsymbol{\tau}_{r} \\
\boldsymbol{J}_{m} \boldsymbol{r}^{-1} \ddot{\boldsymbol{q}}+\boldsymbol{B}_{m} \boldsymbol{r}^{-1} \dot{\boldsymbol{q}}+\boldsymbol{r} \boldsymbol{\tau}_{r}=\boldsymbol{K}_{m} \boldsymbol{I}_{a} \\
\boldsymbol{v}=\boldsymbol{R} \boldsymbol{I}_{a}+\boldsymbol{L} \dot{\boldsymbol{I}}_{a}+\boldsymbol{k}_{b} \boldsymbol{r}^{-1} \dot{\boldsymbol{q}}+\boldsymbol{\varphi}(t)
\end{gathered}
$$

where $\boldsymbol{q} \in R^{n}$ denotes the joint coordinates and is the vector of joint positions, $\boldsymbol{D}(\boldsymbol{q}) \in R^{n \times n}$ is the inertia matrix, $\boldsymbol{C}(\boldsymbol{q}, \dot{\boldsymbol{q}}) \dot{\boldsymbol{q}} \in R^{n}$ the vector of centrifugal and Coriolis torques, $\boldsymbol{G}(\boldsymbol{q}) \in R^{n}$ the vector of gravitational torques, $\boldsymbol{F}_{e} \in R^{m}$ the contact forces vector, $\boldsymbol{\tau}_{r} \in R^{n}$ the input torque vector of robot manipulator. $\boldsymbol{J}_{m}, \boldsymbol{B}_{m}$ and $\boldsymbol{r}$ are the $n \times n$ diagonal matrices for motor coefficients namely the actuator inertia, damping, and reduction gear, respectively. $\boldsymbol{K}_{m} \in R^{n \times n}$ is a diagonal matrix of the torque constants, $\boldsymbol{I}_{a} \in R^{n}$ is the vector of motor currents, $\boldsymbol{v} \in R^{n}$ is the vector of motor voltages and $\boldsymbol{\varphi}(t) \in R^{n}$ is a vector of external disturbances. $\boldsymbol{R}, \boldsymbol{L}$ and $\boldsymbol{K}_{b}$ represent the $n \times n$ diagonal matrices for the coefficients of armature resistance, inductance, and back-emf constant 
respectively. $\boldsymbol{J}(\boldsymbol{q}) \in R^{m \times n}$ is the Jacobian matrix of the robot arms, which converts the joint space to the task space as

$$
\dot{\boldsymbol{x}}=\boldsymbol{J}(\boldsymbol{q}) \dot{\boldsymbol{q}}
$$

where $\boldsymbol{x} \in R^{m}$ is the vector which determines the position of the end-effector. The time derivative of equation (4) can be written as

$$
\ddot{\boldsymbol{x}}=\dot{J}(\boldsymbol{q}) \dot{\boldsymbol{q}}+J(\boldsymbol{q}) \ddot{q}
$$

We consider that $m=n$ and the robot trajectory is never in a singular configuration, i.e., the inverse of the Jacobian matrix always exists. Note that vectors and matrices are represented in bold form for clarity. By considering (1)-(3), one can present the state-space model as

$$
\dot{\boldsymbol{z}}=\boldsymbol{f}(\mathbf{z})+\boldsymbol{b} \boldsymbol{v}-\boldsymbol{b} \boldsymbol{\varphi}(t)
$$

where $\boldsymbol{v}$ is considered as the inputs, $\boldsymbol{z}$ is the state vector and $\boldsymbol{f}(\mathbf{z})$ is of the form

$$
\boldsymbol{f}(\mathbf{z})=\left[\begin{array}{c}
\boldsymbol{z}_{2} \\
\left(\boldsymbol{J}_{m} \boldsymbol{r}^{-1}+\boldsymbol{r D}\left(\mathbf{z}_{1}\right)\right)^{-1} \boldsymbol{W} \\
-\boldsymbol{L}^{-1}\left(\boldsymbol{K}_{b} \boldsymbol{r}^{-1} \boldsymbol{z}_{2}+\boldsymbol{R} \boldsymbol{z}_{3}\right)
\end{array}\right]
$$

in which,

$$
\begin{gathered}
\boldsymbol{W}=-\left(\boldsymbol{B}_{m} \boldsymbol{r}^{-1}+\boldsymbol{r} \boldsymbol{C}\left(\boldsymbol{z}_{1}, \boldsymbol{z}_{2}\right)\right) \mathbf{z}_{2}- \\
\boldsymbol{r} \boldsymbol{g}\left(\boldsymbol{z}_{1}\right)+\boldsymbol{K}_{m} \boldsymbol{z}_{3}-\boldsymbol{r} \boldsymbol{J}^{T} \boldsymbol{F}_{e} \\
\boldsymbol{b}=\left[\begin{array}{c}
0 \\
0 \\
\boldsymbol{L}^{-1}
\end{array}\right], \quad \boldsymbol{z}=\left[\begin{array}{c}
\boldsymbol{q} \\
\dot{\boldsymbol{q}} \\
\boldsymbol{I}_{a}
\end{array}\right]
\end{gathered}
$$

A highly coupled nonlinear system in a non-companion form is shown by the aforementioned state-space equation.

\section{Fuzzy Uncertainty Estimation}

One can represent the voltage equation (3) as

$$
\boldsymbol{v}=\boldsymbol{R} \boldsymbol{I}_{a}+\boldsymbol{k}_{b} \boldsymbol{r}^{-1} \dot{\boldsymbol{q}}+\boldsymbol{\mu}(t)
$$

in which $\boldsymbol{\mu}(t)$ denotes the uncertainty in form of

$$
\boldsymbol{\mu}=\boldsymbol{L} \dot{\boldsymbol{I}}_{a}+\boldsymbol{\varphi}(t)
$$

where $\boldsymbol{L} \dot{\boldsymbol{I}}_{a}$ and $\boldsymbol{\varphi}(t)$ are expressed as un-modeled dynamics and external disturbances respectively. By considering (9), uncertainty can be represented in scalar form as

$$
\boldsymbol{\mu}_{i}=\boldsymbol{v}_{i}(t)-\boldsymbol{R}_{i} \boldsymbol{I}_{i a}-\boldsymbol{k}_{i b} \boldsymbol{r}_{i}^{-1} \dot{\boldsymbol{q}}_{i}
$$

In order to estimate $\boldsymbol{\mu}_{i}$, the fuzzy estimator is employed. The motor current $\boldsymbol{I}_{i a}$ and the joint velocity $\dot{\boldsymbol{q}}_{i}$ and $\boldsymbol{v}_{i}(t-\varepsilon)$ are considered as inputs of fuzzy system. To estimate the uncertainty, $\boldsymbol{v}_{i}(t)$ is not available. Instead, $\boldsymbol{v}_{i}(t-\varepsilon)$, which is the past information of $\boldsymbol{v}_{i}(t)$, is given as the input of the fuzzy system, where $\varepsilon$ is the time delay. By allocating three membership functions named as Positive (P), Zero $(\mathrm{Z})$ and Negative $(\mathrm{N})$ to each fuzzy input, 27 fuzzy rules will cover the whole space. The linguistic rules of fuzzy are utilized in the Mamdani type as

Rule l: If $\boldsymbol{I}_{i a}$ is $A_{l}, \dot{\boldsymbol{q}}_{i}$ is $B_{l}$ and $\boldsymbol{v}_{i}(t-$ $\varepsilon$ ) is $C_{l}$ Then $\hat{\boldsymbol{\mu}}_{i}$ is $D_{l}$

where Rule $l$ represents the $l$-th fuzzy rule for $l=1, \ldots, 27$, fuzzy membership functions $A_{l}, B_{l}, C_{l}$ and $D_{l}$ belong to the fuzzy variables $\boldsymbol{I}_{i a}, \dot{\boldsymbol{q}}_{i}, \boldsymbol{v}_{i}(t-\varepsilon)$ and $\widehat{\boldsymbol{\mu}}_{i}$ respectively. The membership functions for input $\boldsymbol{I}_{i a}$ are given as follows

$$
\begin{gathered}
\mu_{P}\left(\boldsymbol{I}_{i a}\right)=e^{\left(-\left(\boldsymbol{I}_{i a}-1\right)^{2} / 2 \sigma_{f}^{2}\right)} \\
\mu_{Z}\left(\boldsymbol{I}_{i a}\right)=e^{\left(-\boldsymbol{I}_{i a}^{2} / 2 \sigma_{f}^{2}\right)} \\
\mu_{N}\left(\boldsymbol{I}_{i a}\right)=e^{\left(-\left(\boldsymbol{I}_{i a}+1\right)^{2} / 2 \sigma_{f}^{2}\right)}
\end{gathered}
$$

The membership functions for inputs $\dot{\boldsymbol{q}}_{i}$ and $\boldsymbol{v}_{i}(t-\varepsilon)$ are chosen as the same of $\boldsymbol{I}_{i a}$. Also, 27 symmetric Gaussian membership functions are defined for the output $\widehat{\boldsymbol{\mu}}_{i}$ in the form of

$$
\mu_{D_{l}}\left(\widehat{\boldsymbol{\mu}}_{i}\right)=e^{\left(-\left(\widehat{\boldsymbol{\mu}}_{i}-\widehat{\boldsymbol{\psi}}_{i l}\right)^{2} / 0.55\right)}
$$

where $\widehat{\boldsymbol{\psi}}_{i l}$ is the center of fuzzy membership function $D_{l}$. In this paper, $\widehat{\boldsymbol{\psi}}_{i l}$ is adjusted by an adaptive law derived by the stability analysis. Using the product inference engine, singleton fuzzifier, center average defuzzifier and Gaussian membership functions [27], we select the fuzzy system for $\widehat{\boldsymbol{\mu}}_{i}$ as follows

$$
\widehat{\boldsymbol{\mu}}_{i}=\frac{\sum_{l=1}^{27} \mu_{A_{l}}\left(\boldsymbol{I}_{i a}\right) \mu_{B_{l}}\left(\dot{\boldsymbol{q}}_{i}\right) \mu_{C_{l}}\left(\boldsymbol{v}_{i}(t-\varepsilon)\right) \widehat{\boldsymbol{\psi}}_{i l}}{\sum_{l=1}^{27} \mu_{A_{l}}\left(\boldsymbol{I}_{i a}\right) \mu_{B_{l}}\left(\dot{\boldsymbol{q}}_{i}\right) \mu_{C_{l}}\left(\boldsymbol{v}_{i}(t-\varepsilon)\right)}
$$

One can easily denote (17) of the form

$$
\widehat{\boldsymbol{\mu}}_{i}=\sum_{l=1}^{27} \boldsymbol{Y}_{i m l} \widehat{\boldsymbol{\psi}}_{i l}=\boldsymbol{Y}_{i m} \widehat{\boldsymbol{\psi}}_{i}
$$

in which $\boldsymbol{Y}_{i m}=\left[\boldsymbol{Y}_{i m 1}, \ldots, \boldsymbol{Y}_{i m 27}\right]$ is a positive regressive vector defined as

$$
\boldsymbol{Y}_{i m l}=\frac{\mu_{A_{l}}\left(\boldsymbol{I}_{i a}\right) \mu_{B_{l}}\left(\dot{\boldsymbol{q}}_{i}\right) \mu_{C_{l}}\left(v_{i}(t-\varepsilon)\right)}{\sum_{l=1}^{27} \mu_{A_{l}}\left(\boldsymbol{I}_{i a}\right) \mu_{B_{l}}\left(\dot{\boldsymbol{q}}_{i}\right) \mu_{C_{l}}\left(\boldsymbol{v}_{i}(t-\varepsilon)\right)}
$$

And $\widehat{\boldsymbol{\psi}}_{i}$ is an adaptive gain vector of the form 


$$
\widehat{\boldsymbol{\psi}}_{i}=\left[\widehat{\boldsymbol{\psi}}_{i 1}, \ldots, \widehat{\boldsymbol{\psi}}_{i 27}\right]^{T}
$$

\section{Impedance Design}

The contact forces vector, $\boldsymbol{F}_{e}$, can be presented as

$$
\boldsymbol{M}_{e} \ddot{\boldsymbol{x}}+\boldsymbol{B}_{e} \dot{\boldsymbol{x}}+\boldsymbol{K}_{e}\left(\boldsymbol{x}-\boldsymbol{x}_{e}\right)=\boldsymbol{F}_{e}
$$

where $\boldsymbol{M}_{e} \in R^{m \times m}, \boldsymbol{B}_{e} \in R^{m \times m}$ and $\boldsymbol{K}_{e} \in R^{m \times m}$ are represented the inertia, damping and stiffness of the environment involved in contact and $\boldsymbol{x}_{e} \in R^{m}$ is the environment equilibrium position in the absence of the contact force. One can take the Laplace transform from both sides of equation (21) to yield

$$
s \boldsymbol{Z}_{e}(s) \boldsymbol{x}(s)-\boldsymbol{k}_{e} \boldsymbol{x}_{e}(s)=\boldsymbol{F}_{e}(s)
$$

in which $\boldsymbol{Z}_{e}(s)$ is defined as

$$
\boldsymbol{Z}_{e}(s)=\boldsymbol{M}_{e} s+\boldsymbol{B}_{e}+\frac{\boldsymbol{K}_{e}}{s}
$$

In contact with the environment, which is a primary goal for impedance control of robotic system, a desired dynamical behavior is defined in Laplace form as

$$
s \boldsymbol{Z}_{R}(s)\left(\boldsymbol{x}_{d}(s)-\boldsymbol{x}_{m}(s)\right)=\boldsymbol{F}_{e}(s)
$$

where $\boldsymbol{x}_{\boldsymbol{d}}$ is the desired trajectory in task-space, $\boldsymbol{x}_{m}$ is the output of reference model and $\boldsymbol{Z}_{R}(s)$ is a desired impedance given as follows

$$
\boldsymbol{Z}_{R}(s)=\boldsymbol{M}_{R} s+\boldsymbol{B}_{R}+\frac{\boldsymbol{K}_{R}}{s}
$$

where $\boldsymbol{M}_{R}, \boldsymbol{B}_{R}$ and $\boldsymbol{K}_{R}$ represent the diagonal $n \times n$ inertia, damping and stiffness of the reference model matrices, respectively. Using (25), equation (24) can be rewritten in scalar form as

$\left(\boldsymbol{M}_{R i} s^{2}+\boldsymbol{B}_{R i} s+\boldsymbol{K}_{R i}\right)\left(\boldsymbol{x}_{d i}(s)-\boldsymbol{x}_{m i}(s)\right)=\boldsymbol{F}_{e i}(s)$

Remark 1. If a robot has no contact with an environment, i.e. $\boldsymbol{F}_{e i}=0$, one can choose proper values for $\boldsymbol{M}_{R i}, \boldsymbol{B}_{R i}$ and $\boldsymbol{K}_{R i}$ such that all roots of polynomial $d(s) \triangleq \boldsymbol{M}_{R i} s^{2}+\boldsymbol{B}_{R i} s+\boldsymbol{K}_{R i}$ are in the open left- half of complex plane, i.e. strictly Hurwitz. Thus, as $t \rightarrow \infty$, $\boldsymbol{x}_{m i} \rightarrow \boldsymbol{x}_{d i}$. This means that the impedance controller behaves as a pure position control (e.g. a path planning problem using the feedback-based compositional rule of inference [28] and a Taylor series tracking control [29]).

Remark 2. If a robotic system interacts with an environment, i.e. $\boldsymbol{F}_{e i} \neq 0$, there exists a difference between $\boldsymbol{x}_{m i}$ and $\boldsymbol{x}_{d i}$ and thus the existing contact force is regulated to protect robot and environment against serious damages.
Remark 3. As a result, the impedance control is designed in such a way that the output of the system follows the output of the reference model (i.e. $\boldsymbol{x} \rightarrow \boldsymbol{x}_{m}$ ). It is worth noting that in order to protect both the robot and the environment, the desired tracking deviation occurs since the reference model performs like a desired dynamical behavior of robotic system in contact with the environment.

\section{Robust Control Design and Stability Analysis}

In section 5, our control objective is to propose an impedance model-reference controller to reduce the difference between the task-space desired trajectory and the desired impedance model. The proposed controller is equipped with fuzzy estimator in order to be robust against the uncertainty including un-modelled dynamics and external disturbances as well as a robustifying term to improve the performance of controller. The block diagram of control system is shown in Fig.1.

The dynamic equation of electrically driven robot manipulators in task-space can be obtained by substituting equation (1) to (2) and using equations (4), and (5) and (9) as follows

$$
\boldsymbol{D}_{x} \ddot{\boldsymbol{x}}+\boldsymbol{C}_{x} \dot{\boldsymbol{x}}+\boldsymbol{G}_{x}+\boldsymbol{R} \boldsymbol{k}_{m}^{-1} \boldsymbol{r} \boldsymbol{J}^{T} \boldsymbol{F}_{e}=\boldsymbol{v}-\boldsymbol{\mu}
$$

where $\boldsymbol{D}_{x}, \boldsymbol{C}_{x}$ and $\boldsymbol{G}_{x}$ are given as

$$
\boldsymbol{D}_{x}=\boldsymbol{R} \boldsymbol{k}_{m}^{-1}\left(\boldsymbol{J}_{m} \boldsymbol{r}^{-1}+\boldsymbol{r D}(\boldsymbol{q})\right) \boldsymbol{J}^{-1}
$$

$\boldsymbol{C}_{x}=\boldsymbol{R} \boldsymbol{k}_{m}^{-1}\left(\boldsymbol{B}_{m} \boldsymbol{r}^{-1}+\boldsymbol{r} \boldsymbol{C}(\boldsymbol{q}, \dot{\boldsymbol{q}})+\boldsymbol{k}_{m} \boldsymbol{R}^{-1} \boldsymbol{k}_{b} \boldsymbol{r}^{-1}-\right.$

$\left.\left(\boldsymbol{J}_{m} \boldsymbol{r}^{-1}+\boldsymbol{r D}(\boldsymbol{q})\right) \boldsymbol{J}^{-1} \dot{\boldsymbol{J}}\right) \boldsymbol{J}^{-1}$

$$
\boldsymbol{G}_{x}=\boldsymbol{R} \boldsymbol{k}_{m}^{-1} \boldsymbol{r} \boldsymbol{G}(\boldsymbol{q})
$$

Some properties of the dynamic equation of electrically driven robot manipulators (Eq. 27) can be expressed as follows [26]:

Property 1: The matrix $\boldsymbol{D}_{x}$ is a symmetric positive definite matrix for all $x \in R^{m}$.

Property 2: The skew-symmetric properties is preserved for $\dot{\boldsymbol{D}}_{x}-2 \boldsymbol{C}_{x}$ so that

$$
\boldsymbol{w}^{T} \dot{\boldsymbol{D}}_{x} \boldsymbol{w}=2 \boldsymbol{w}^{T} \boldsymbol{C}_{x} \boldsymbol{w}, \quad \forall \boldsymbol{w} \in R^{m}
$$

Property 3: The task-space dynamic equation can also be linearly parameterized with using a suitable selected parameter vector $\overline{\boldsymbol{P}}$ and a known regression matrix $\overline{\boldsymbol{Y}}(\boldsymbol{x}, \dot{\boldsymbol{x}}, \ddot{\boldsymbol{x}})$, i.e.

$$
D_{x} \ddot{\boldsymbol{x}}+\boldsymbol{C}_{x} \dot{\boldsymbol{x}}+\boldsymbol{G}_{x}=\overline{\boldsymbol{Y}}(\boldsymbol{x}, \dot{\boldsymbol{x}}, \ddot{\boldsymbol{x}}) \overline{\boldsymbol{P}}
$$




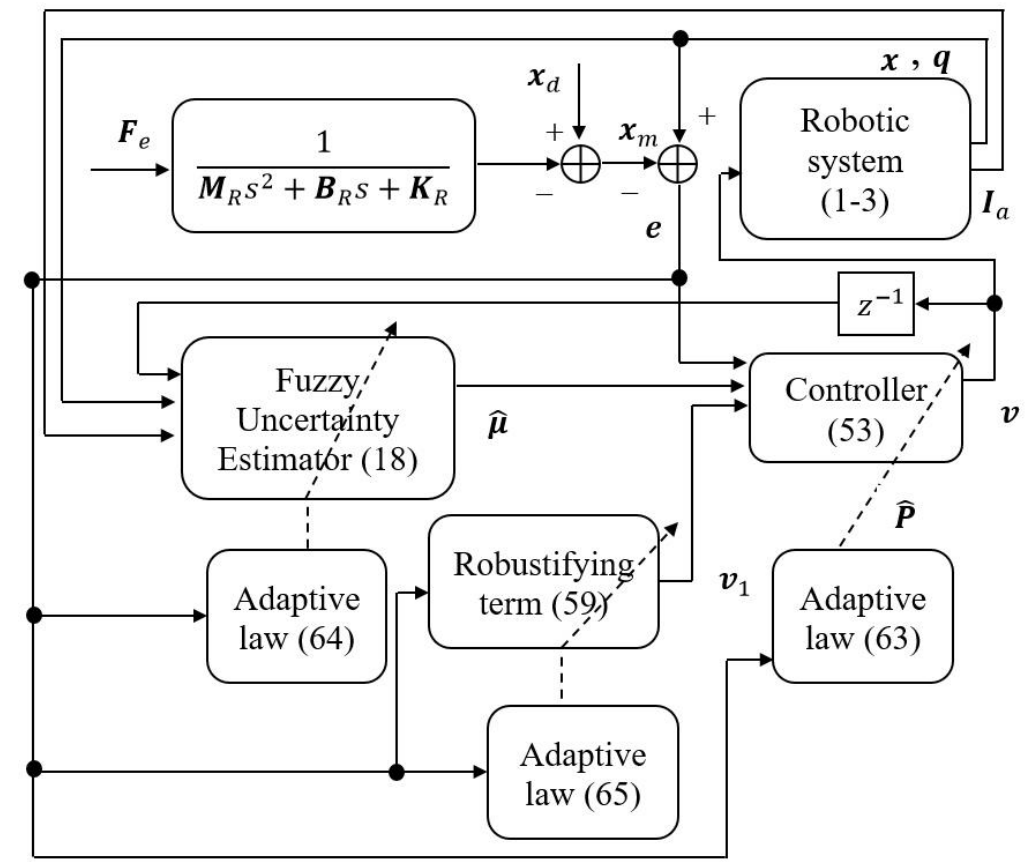

Fig. 1. The Block diagram of the control system

Let us define the error dynamics as

$$
\Xi=\dot{e}+\boldsymbol{k}_{1} \boldsymbol{e}
$$

in which $\boldsymbol{k}_{1}$ is the $n \times n$ constant positive definite matrix and $\boldsymbol{e}$ is the reference error which is the difference between the output of the closed loop system and the reference model output expressed by

$$
\boldsymbol{e}=\boldsymbol{x}-\boldsymbol{x}_{m}
$$

Consider a following positive definite function as

$V=\frac{1}{2} \boldsymbol{\Xi}^{T} \boldsymbol{D}_{x} \boldsymbol{\Xi}+\frac{1}{2}(\widehat{\boldsymbol{P}}-\boldsymbol{P})^{T} \boldsymbol{\Gamma}^{-1}(\widehat{\boldsymbol{P}}-\boldsymbol{P})+\frac{1}{2 \gamma_{\eta}}(\hat{\eta}-$

$\eta)^{2}+\frac{1}{2 \gamma_{f}}(\widehat{\boldsymbol{\psi}}-\boldsymbol{\psi})^{T}(\widehat{\boldsymbol{\psi}}-\boldsymbol{\psi})$

Taking the time derivative of $V$ gives that

$\dot{V}=\boldsymbol{\Xi}^{T} \boldsymbol{D}_{x} \dot{\boldsymbol{\Xi}}+\frac{1}{2} \boldsymbol{\Xi}^{T} \dot{\boldsymbol{D}}_{x} \boldsymbol{\Xi}+\dot{\hat{\boldsymbol{P}}}^{T} \boldsymbol{\Gamma}^{-1}(\widehat{\boldsymbol{P}}-\boldsymbol{P})+\frac{1}{\gamma_{\eta}} \dot{\hat{\eta}}(\hat{\eta}-$

$\eta)+\frac{1}{\gamma_{f}} \dot{\boldsymbol{\psi}}^{T}(\widehat{\boldsymbol{\psi}}-\boldsymbol{\psi})$

Using (33) and (34), equation (36) yields

$$
\begin{aligned}
& \dot{V}=\boldsymbol{\Xi}^{T} \boldsymbol{D}_{x}\left(\ddot{\boldsymbol{x}}-\ddot{\boldsymbol{x}}_{m}+\boldsymbol{k}_{1} \dot{\boldsymbol{e}}\right)+\frac{1}{2} \boldsymbol{\Xi}^{T} \dot{\boldsymbol{D}}_{x} \boldsymbol{\Xi}+\dot{\widehat{\boldsymbol{P}}}^{T} \boldsymbol{\Gamma}^{-1}(\widehat{\boldsymbol{P}}- \\
& \boldsymbol{P})+\frac{1}{\gamma_{\eta}} \dot{\hat{\eta}}(\hat{\eta}-\eta)+\frac{1}{\gamma_{f}} \dot{\boldsymbol{\psi}}^{T}(\widehat{\boldsymbol{\psi}}-\boldsymbol{\psi}) \\
& \dot{V}=\boldsymbol{\Xi}^{T}\left(\boldsymbol{D}_{x} \ddot{\boldsymbol{x}}-\boldsymbol{D}_{x}\left(\ddot{\boldsymbol{x}}_{m}-\boldsymbol{k}_{1} \dot{\boldsymbol{e}}\right)\right)+\frac{1}{2} \boldsymbol{\Xi}^{T} \dot{\boldsymbol{D}}_{x} \boldsymbol{\Xi}+ \\
& \dot{\boldsymbol{P}}^{T} \boldsymbol{\Gamma}^{-1}(\widehat{\boldsymbol{P}}-\boldsymbol{P})+\frac{1}{\gamma_{\eta}} \dot{\hat{\eta}}(\hat{\eta}-\eta)+\frac{1}{\gamma_{f}} \dot{\boldsymbol{\boldsymbol { \psi }}}^{T}(\widehat{\boldsymbol{\psi}}-\boldsymbol{\psi})
\end{aligned}
$$

One can rewrite equation (27) as

$$
\boldsymbol{D}_{x} \ddot{\boldsymbol{x}}=\boldsymbol{v}-\boldsymbol{\mu}-\boldsymbol{C}_{x} \dot{\boldsymbol{x}}-\boldsymbol{G}_{x}-\boldsymbol{R} \boldsymbol{k}_{m}^{-1} \boldsymbol{r} \boldsymbol{J}^{T} \boldsymbol{F}_{e}
$$

Substituting (39) into (38) we have

$$
\begin{aligned}
& \dot{V}=\Xi^{T}\left(\boldsymbol{v}-\boldsymbol{\mu}-\boldsymbol{C}_{x} \dot{\boldsymbol{x}}-\boldsymbol{G}_{x}-\boldsymbol{R} \boldsymbol{k}_{m}^{-1} \boldsymbol{r} \boldsymbol{J}^{T} \boldsymbol{F}_{e}-\boldsymbol{D}_{x}\left(\ddot{\boldsymbol{x}}_{m}-\right.\right. \\
& \left.\left.\boldsymbol{k}_{1} \dot{\boldsymbol{e}}\right)\right)+\frac{1}{2} \boldsymbol{\Xi}^{T} \dot{\boldsymbol{D}}_{x} \boldsymbol{\Xi}+\dot{\widehat{\boldsymbol{P}}}^{T} \boldsymbol{\Gamma}^{-1}(\widehat{\boldsymbol{P}}-\boldsymbol{P})+\frac{1}{\gamma_{\eta}} \dot{\hat{\eta}}(\hat{\eta}-\eta)+ \\
& \frac{1}{\gamma_{f}} \dot{\boldsymbol{\boldsymbol { \psi }}}^{T}(\widehat{\boldsymbol{\psi}}-\boldsymbol{\psi})
\end{aligned}
$$

Considering (33) and (34), one can obtain

$$
\dot{\boldsymbol{x}}=\boldsymbol{\Xi}+\dot{\boldsymbol{x}}_{m}-\boldsymbol{k}_{1} \boldsymbol{e}
$$

Thus, substituting (41) into (40) yields to 
$\dot{V}=\boldsymbol{\Xi}^{T}\left(\boldsymbol{v}-\boldsymbol{\mu}-\boldsymbol{C}_{x}\left(\boldsymbol{\Xi}+\dot{\boldsymbol{x}}_{m}-\boldsymbol{k}_{1} \boldsymbol{e}\right)-\boldsymbol{G}_{x}-\right.$

$\left.\boldsymbol{R} \boldsymbol{k}_{m}^{-1} \boldsymbol{r} \boldsymbol{J}^{T} \boldsymbol{F}_{e}-\boldsymbol{D}_{x}\left(\ddot{\boldsymbol{x}}_{m}-\boldsymbol{k}_{1} \dot{\boldsymbol{e}}\right)\right)+\frac{1}{2} \boldsymbol{\Xi}^{T} \dot{\boldsymbol{D}}_{x} \boldsymbol{\Xi}+$

$\dot{\hat{\boldsymbol{P}}}^{T} \boldsymbol{\Gamma}^{-1}(\widehat{\boldsymbol{P}}-\boldsymbol{P})+\frac{1}{\gamma_{\eta}} \dot{\hat{\eta}}(\hat{\eta}-\eta)+\frac{1}{\gamma_{f}} \dot{\boldsymbol{\psi}}^{T}(\widehat{\boldsymbol{\psi}}-\boldsymbol{\psi})$

In other words,

$\dot{V}=\frac{1}{2} \boldsymbol{\Xi}^{T}\left(\dot{\boldsymbol{D}}_{x}-2 \boldsymbol{C}_{x}\right) \boldsymbol{\Xi}+\boldsymbol{\Xi}^{T}\left(\boldsymbol{v}-\boldsymbol{\mu}-\boldsymbol{D}_{x}\left(\ddot{\boldsymbol{x}}_{m}-\boldsymbol{k}_{1} \dot{\boldsymbol{e}}\right)-\right.$

$\left.\boldsymbol{C}_{x}\left(\dot{\boldsymbol{x}}_{m}-\boldsymbol{k}_{1} \boldsymbol{e}\right)-\boldsymbol{G}_{x}-\boldsymbol{R} \boldsymbol{k}_{m}^{-1} \boldsymbol{r} \boldsymbol{J}^{T} \boldsymbol{F}_{e}\right)+\dot{\widehat{\boldsymbol{P}}}^{T} \boldsymbol{\Gamma}^{-1}(\widehat{\boldsymbol{P}}-$

$\boldsymbol{P})+\frac{1}{\gamma_{\eta}} \dot{\hat{\eta}}(\hat{\eta}-\eta)+\frac{1}{\gamma_{f}} \dot{\hat{\boldsymbol{\psi}}}^{T}(\widehat{\boldsymbol{\psi}}-\boldsymbol{\psi})$

Let us define the modified velocity and acceleration in task-space as follows

$$
\begin{aligned}
& \dot{\boldsymbol{x}}_{r}=\dot{\boldsymbol{x}}_{m}-\boldsymbol{k}_{1} \boldsymbol{e} \\
& \ddot{\boldsymbol{x}}_{r}=\ddot{\boldsymbol{x}}_{m}-\boldsymbol{k}_{1} \dot{\boldsymbol{e}}
\end{aligned}
$$

By using the skew-symmetric property (31) and equations (44) and (45), one can modify (43) as

$\dot{V}=\boldsymbol{\Xi}^{T}\left(\boldsymbol{v}-\boldsymbol{\mu}-\boldsymbol{D}_{x} \ddot{\boldsymbol{x}}_{r}-\boldsymbol{C}_{x} \dot{\boldsymbol{x}}_{r}-\boldsymbol{G}_{x}-\boldsymbol{R} \boldsymbol{k}_{m}^{-1} \boldsymbol{r} \boldsymbol{J}^{T} \boldsymbol{F}_{e}\right)+$ $\dot{\hat{\boldsymbol{P}}}^{T} \boldsymbol{\Gamma}^{-1}(\widehat{\boldsymbol{P}}-\boldsymbol{P})+\frac{1}{\gamma_{\eta}} \dot{\hat{\eta}}(\hat{\eta}-\eta)+\frac{1}{\gamma_{f}} \dot{\hat{\boldsymbol{\psi}}}^{T}(\widehat{\boldsymbol{\psi}}-\boldsymbol{\psi})$

Considering linear parameterization property (32), we can write

$\boldsymbol{D}_{x} \ddot{\boldsymbol{x}}_{r}+\boldsymbol{C}_{x} \dot{\boldsymbol{x}}_{r}+\boldsymbol{G}_{x}=\boldsymbol{Y}_{x}\left(\boldsymbol{x}, \dot{\boldsymbol{x}}, \dot{\boldsymbol{x}}_{r}, \ddot{\boldsymbol{x}}_{r}\right) \boldsymbol{P}_{r}$

It is noted that $\boldsymbol{Y}_{x}\left(\boldsymbol{x}, \dot{\boldsymbol{x}}, \dot{\boldsymbol{x}}_{r}, \ddot{\boldsymbol{x}}_{r}\right) \in R^{n \times n_{p}}$ and $\boldsymbol{P}_{r} \in R^{n_{p} \times 1}$ are regression matrix and vector of parameters, respectively, where $n_{p}$ is the number of parameters. By substituting (47) into (46),

$\dot{V}=\boldsymbol{\Xi}^{T}\left(\boldsymbol{v}-\boldsymbol{\mu}-\boldsymbol{Y}_{x}\left(\boldsymbol{x}, \dot{\boldsymbol{x}}, \dot{\boldsymbol{x}}_{r}, \ddot{\boldsymbol{x}}_{r}\right) \boldsymbol{P}_{r}-\boldsymbol{R} \boldsymbol{k}_{m}^{-1} \boldsymbol{r} \boldsymbol{J}^{T} \boldsymbol{F}_{e}\right)+$

$\dot{\hat{\boldsymbol{P}}}^{T} \boldsymbol{\Gamma}^{-1}(\widehat{\boldsymbol{P}}-\boldsymbol{P})+\frac{1}{\gamma_{\eta}} \dot{\hat{\eta}}(\hat{\eta}-\eta)+\frac{1}{\gamma_{f}} \dot{\boldsymbol{\psi}}^{T}(\widehat{\boldsymbol{\psi}}-\boldsymbol{\psi})$

Remark 4. Researchers have faced difficulties in analyzing the motion equation in task-space since the exact kinematics model is not available in practice or surplus of sensors is required to monitor the motion of an end-effector. To deal this problem, let us consider the transformation functions as follows

$$
\begin{gathered}
\dot{\boldsymbol{x}}_{r}=\boldsymbol{J}(\boldsymbol{q}) \dot{\boldsymbol{q}}_{r} \\
\ddot{\boldsymbol{x}}_{r}=\dot{\boldsymbol{J}}(\boldsymbol{q}) \dot{\boldsymbol{q}}_{r}+\boldsymbol{J}(\boldsymbol{q}) \ddot{\boldsymbol{q}}_{r}
\end{gathered}
$$

By applying (49) and (50) to equation (47) and using (28-30), it can be obtained

$$
\boldsymbol{Y}_{x}\left(\boldsymbol{x}, \dot{\boldsymbol{x}}, \dot{\boldsymbol{x}}_{r}, \ddot{\boldsymbol{x}}_{r}\right) \boldsymbol{P}_{r}=\boldsymbol{Y}\left(\boldsymbol{q}, \dot{\boldsymbol{q}}, \dot{\boldsymbol{q}}_{r}, \ddot{\boldsymbol{q}}_{r}\right) \boldsymbol{P}
$$

Using (51) and also transforming the error dynamics (33) into joint space, i.e. $\boldsymbol{\Xi}=\boldsymbol{J}\left(\dot{\boldsymbol{q}}-\dot{\boldsymbol{q}}_{r}\right)$, equation (48) can be modified as

$$
\begin{aligned}
& \dot{V}=\left(\boldsymbol{J}\left(\dot{\boldsymbol{q}}-\dot{\boldsymbol{q}}_{r}\right)\right)^{T}\left(\boldsymbol{v}-\boldsymbol{\mu}-\boldsymbol{Y}\left(\boldsymbol{q}, \dot{\boldsymbol{q}}, \dot{\boldsymbol{q}}_{r}, \ddot{\boldsymbol{q}}_{r}\right) \boldsymbol{P}-\right. \\
& \left.\boldsymbol{R} \boldsymbol{k}_{m}^{-1} \boldsymbol{r} \boldsymbol{J}^{T} \boldsymbol{F}_{e}\right)+\dot{\boldsymbol{P}}^{T} \boldsymbol{\Gamma}^{-1}(\widehat{\boldsymbol{P}}-\boldsymbol{P})+\frac{1}{\gamma_{\eta}} \dot{\hat{\hat{\eta}}}(\hat{\eta}-\eta)+ \\
& \frac{1}{\gamma_{f}} \dot{\boldsymbol{\boldsymbol { \psi }}}^{T}(\widehat{\boldsymbol{\psi}}-\boldsymbol{\psi})
\end{aligned}
$$

This paper proposes the control law as

$\boldsymbol{v}=\boldsymbol{Y}\left(\boldsymbol{q}, \dot{\boldsymbol{q}}, \dot{\boldsymbol{q}}_{r}, \ddot{\boldsymbol{q}}_{r}\right) \widehat{\boldsymbol{P}}-\boldsymbol{k}_{2} \boldsymbol{J}\left(\dot{\boldsymbol{q}}-\dot{\boldsymbol{q}}_{r}\right)+\widehat{\boldsymbol{\mu}}-\boldsymbol{v}_{1}$

in which $\boldsymbol{k}_{2} \in R^{n \times n}$ is a diagonal positive definite matrix and $\boldsymbol{v}_{1}$ is a robustifying term. By applying the control law (53) to (52), we have

$\dot{V}=\left(\boldsymbol{J}\left(\dot{\boldsymbol{q}}-\dot{\boldsymbol{q}}_{r}\right)\right)^{T}\left(\boldsymbol{Y}\left(\boldsymbol{q}, \dot{\boldsymbol{q}}, \dot{\boldsymbol{q}}_{r}, \ddot{\boldsymbol{q}}_{r}\right)(\widehat{\boldsymbol{P}}-\boldsymbol{P})-\boldsymbol{k}_{2} \boldsymbol{J}(\dot{\boldsymbol{q}}-\right.$

$\left.\left.\dot{\boldsymbol{q}}_{r}\right)+\widehat{\boldsymbol{\mu}}-\boldsymbol{\mu}-\boldsymbol{R} \boldsymbol{k}_{m}^{-1} \boldsymbol{r} \boldsymbol{J}^{T} \boldsymbol{F}_{e}-\boldsymbol{v}_{1}\right)+\dot{\widehat{\boldsymbol{P}}}^{T} \boldsymbol{\Gamma}^{-1}(\widehat{\boldsymbol{P}}-\boldsymbol{P})+$

$\frac{1}{\gamma_{\eta}} \dot{\hat{\eta}}(\hat{\eta}-\eta)+\frac{1}{\gamma_{f}} \dot{\boldsymbol{\psi}}^{T}(\widehat{\boldsymbol{\psi}}-\boldsymbol{\psi})$

Assume that $\boldsymbol{R} \boldsymbol{k}_{m}^{-1} \boldsymbol{r}$ and $\boldsymbol{F}_{e}$ are bounded as follows

$$
\begin{gathered}
\left\|\boldsymbol{R} \boldsymbol{k}_{m}^{-1} \boldsymbol{r}\right\| \leq \eta_{1} \\
\left\|\boldsymbol{F}_{e}\right\| \leq \eta_{2}
\end{gathered}
$$

Using the Cauchy-Schwartz inequality, we have

$$
\left\|\left(\boldsymbol{J}\left(\dot{\boldsymbol{q}}-\dot{\boldsymbol{q}}_{r}\right)\right)^{T} \boldsymbol{R} \boldsymbol{k}_{m}^{-1} \boldsymbol{r} \boldsymbol{J}^{T} \boldsymbol{F}_{e}\right\| \leq \eta\left\|\left(\boldsymbol{J}\left(\dot{\boldsymbol{q}}-\dot{\boldsymbol{q}}_{r}\right)\right)^{T} \boldsymbol{J}^{T}\right\|
$$

where $\eta \triangleq \eta_{1} \eta_{2}$. By considering (57), equation (54) can be written as

$$
\begin{aligned}
& \dot{V} \leq\left(\boldsymbol{J}\left(\dot{\boldsymbol{q}}-\dot{\boldsymbol{q}}_{r}\right)\right)^{T}\left(\boldsymbol{Y}\left(\boldsymbol{q}, \dot{\boldsymbol{q}}_{,} \dot{\boldsymbol{q}}_{r}, \ddot{\boldsymbol{q}}_{r}\right)(\widehat{\boldsymbol{P}}-\boldsymbol{P})-\boldsymbol{k}_{2} \boldsymbol{J}(\dot{\boldsymbol{q}}-\right. \\
& \left.\left.\dot{\boldsymbol{q}}_{r}\right)+\widehat{\boldsymbol{\mu}}-\boldsymbol{\mu}-\boldsymbol{v}_{1}\right)+\eta\left\|\left(\boldsymbol{J}\left(\dot{\boldsymbol{q}}-\dot{\boldsymbol{q}}_{r}\right)\right)^{T} \boldsymbol{J}^{T}\right\|+ \\
& \dot{\widehat{\boldsymbol{P}}}^{T} \boldsymbol{\Gamma}^{-1}(\widehat{\boldsymbol{P}}-\boldsymbol{P})+\frac{1}{\gamma_{\eta}} \dot{\hat{\eta}}(\hat{\eta}-\eta)+\frac{1}{\gamma_{f}} \dot{\hat{\boldsymbol{\psi}}}^{T}(\widehat{\boldsymbol{\psi}}-\boldsymbol{\psi})
\end{aligned}
$$

The robustifying term is selected as

$$
\boldsymbol{v}_{1}=\frac{\widehat{\eta}^{2} \boldsymbol{J}^{T} \boldsymbol{J}^{2}\left(\dot{\boldsymbol{q}}-\dot{\boldsymbol{q}}_{r}\right)}{\left\|\left(\boldsymbol{J}\left(\dot{\boldsymbol{q}}-\dot{\boldsymbol{q}}_{r}\right)\right)^{T} \boldsymbol{J}^{T}\right\| \hat{\eta}+\delta e^{-\sigma t}}
$$

By substituting (59) in (58) and some manipulations, we have

$$
\begin{aligned}
& \dot{V} \leq\left(\boldsymbol{J}\left(\dot{\boldsymbol{q}}-\dot{\boldsymbol{q}}_{r}\right)\right)^{T}\left(\boldsymbol{Y}\left(\boldsymbol{q}, \dot{\boldsymbol{q}}, \dot{\boldsymbol{q}}_{r}, \ddot{\boldsymbol{q}}_{r}\right)(\widehat{\boldsymbol{P}}-\boldsymbol{P})-\boldsymbol{k}_{2} \boldsymbol{J}(\dot{\boldsymbol{q}}-\right. \\
& \left.\left.\dot{\boldsymbol{q}}_{r}\right)+\widehat{\boldsymbol{\mu}}-\boldsymbol{\mu}\right)-(\hat{\eta}-\eta)\left\|\left(\boldsymbol{J}\left(\dot{\boldsymbol{q}}-\dot{\boldsymbol{q}}_{r}\right)\right)^{T} \boldsymbol{J}^{T}\right\|+\delta e^{-\sigma t}+ \\
& \dot{\widehat{\boldsymbol{P}}}^{T} \boldsymbol{\Gamma}^{-1}(\widehat{\boldsymbol{P}}-\boldsymbol{P})+\frac{1}{\gamma_{\eta}} \dot{\hat{\eta}}(\hat{\eta}-\eta)+\frac{1}{\gamma_{f}} \dot{\boldsymbol{\boldsymbol { \psi }}}^{T}(\widehat{\boldsymbol{\psi}}-\boldsymbol{\psi})
\end{aligned}
$$

Assume that the uncertainty (10) can be modelled as

$$
\boldsymbol{\mu}=\boldsymbol{Y}_{m} \boldsymbol{\psi}+\boldsymbol{\varepsilon}_{f}
$$

where $\boldsymbol{\varepsilon}_{f}$ is a modelling error. One can employ (18) and (61) to (60) obtaining 
$\dot{V} \leq\left(\boldsymbol{J}\left(\dot{\boldsymbol{q}}-\dot{\boldsymbol{q}}_{r}\right)\right)^{T}\left(\boldsymbol{Y}\left(\boldsymbol{q}, \dot{\boldsymbol{q}}, \dot{\boldsymbol{q}}_{r}, \ddot{\boldsymbol{q}}_{r}\right)(\widehat{\boldsymbol{P}}-\boldsymbol{P})-\boldsymbol{k}_{2} \boldsymbol{J}(\dot{\boldsymbol{q}}-\right.$ $\left.\left.\dot{\boldsymbol{q}}_{r}\right)\right)+\left(\boldsymbol{J}\left(\dot{\boldsymbol{q}}-\dot{\boldsymbol{q}}_{r}\right)\right)^{T} \boldsymbol{Y}_{m}(\widehat{\boldsymbol{\psi}}-\boldsymbol{\psi})-\left(\boldsymbol{J}\left(\dot{\boldsymbol{q}}-\dot{\boldsymbol{q}}_{r}\right)\right)^{T} \boldsymbol{\varepsilon}_{f}+$ $\delta e^{-\sigma t}-(\hat{\eta}-\eta)\left\|\left(\boldsymbol{J}\left(\dot{\boldsymbol{q}}-\dot{\boldsymbol{q}}_{r}\right)\right)^{T} \boldsymbol{J}^{T}\right\|+\dot{\widehat{\boldsymbol{P}}}^{T} \boldsymbol{\Gamma}^{-1}(\widehat{\boldsymbol{P}}-$

$\boldsymbol{P})+\frac{1}{\gamma_{\eta}} \dot{\hat{\eta}}(\hat{\eta}-\eta)+\frac{1}{\gamma_{f}} \dot{\boldsymbol{\psi}}^{T}(\hat{\boldsymbol{\psi}}-\boldsymbol{\psi})$

If the adaptation laws are chosen as

$$
\begin{gathered}
\dot{\overrightarrow{\boldsymbol{P}}}^{T}=-\left(\boldsymbol{J}\left(\dot{\boldsymbol{q}}-\dot{\boldsymbol{q}}_{r}\right)\right)^{T} \boldsymbol{Y}\left(\boldsymbol{q}, \dot{\boldsymbol{q}}, \dot{\boldsymbol{q}}_{r}, \ddot{\boldsymbol{q}}_{r}\right) \boldsymbol{\Gamma} \\
\dot{\boldsymbol{\boldsymbol { \psi }}}^{T}=-\gamma_{f}\left(\boldsymbol{J}\left(\dot{\boldsymbol{q}}-\dot{\boldsymbol{q}}_{r}\right)\right)^{T} \boldsymbol{Y}_{m} \\
\dot{\hat{\eta}}=\gamma_{\eta}\left\|\left(\boldsymbol{J}\left(\dot{\boldsymbol{q}}-\dot{\boldsymbol{q}}_{r}\right)\right)^{T} \boldsymbol{J}^{T}\right\|
\end{gathered}
$$

Then equation (62) is expressed as

$\dot{V} \leq-\left(\boldsymbol{J}\left(\dot{\boldsymbol{q}}-\dot{\boldsymbol{q}}_{r}\right)\right)^{T} \boldsymbol{k}_{2} \boldsymbol{J}\left(\dot{\boldsymbol{q}}-\dot{\boldsymbol{q}}_{r}\right)-\left(\boldsymbol{J}\left(\dot{\boldsymbol{q}}-\dot{\boldsymbol{q}}_{r}\right)\right)^{T} \boldsymbol{\varepsilon}_{f}+$ $\delta e^{-\sigma t}$

The following assumptions are made to complete the stability analysis $[26,30]$ :

Assumption 1 The desired trajectory $\boldsymbol{x}_{d}$ must be smooth in the sense that $\boldsymbol{x}_{d}$ and its derivatives up to a necessary order are available and all uniformly bounded.

Assumption 2 The external disturbance $\boldsymbol{\varphi}(t)$ is bounded as $\|\varphi(t)\| \leq \varphi_{\max }$.

where $\varphi_{\max }$ is the maximum value for the external disturbance. The electric motors should be protected against over-voltage, thus the next assumption is considered.

Assumption 3 The motor voltage is bounded as $\|\boldsymbol{v}(t)\| \leq v_{\text {max }}$.

The error dynamics, $\boldsymbol{\Xi}=\boldsymbol{J}\left(\dot{\boldsymbol{q}}-\dot{\boldsymbol{q}}_{r}\right)$, reduces if $\dot{V}<0$. Hence, satisfying $\dot{V}<0$ for equation (66) yields

$-\left(\boldsymbol{J}\left(\dot{\boldsymbol{q}}-\dot{\boldsymbol{q}}_{r}\right)\right)^{T} \boldsymbol{\varepsilon}_{f}+\delta e^{-\sigma t} \leq\left(\boldsymbol{J}\left(\dot{\boldsymbol{q}}-\dot{\boldsymbol{q}}_{r}\right)\right)^{T} \boldsymbol{k}_{2} \boldsymbol{J}(\dot{\boldsymbol{q}}-$ $\left.\dot{\boldsymbol{q}}_{r}\right)$

Since $\boldsymbol{k}_{2}$ is a positive definite matrix, then the righthand side of (67) is bounded as

$\lambda_{\text {min }}\left(\boldsymbol{k}_{2}\right)\left\|\boldsymbol{J}\left(\dot{\boldsymbol{q}}-\dot{\boldsymbol{q}}_{r}\right)\right\|^{2} \leq\left(\boldsymbol{J}\left(\dot{\boldsymbol{q}}-\dot{\boldsymbol{q}}_{r}\right)\right)^{T} \boldsymbol{k}_{2} \boldsymbol{J}\left(\dot{\boldsymbol{q}}-\dot{\boldsymbol{q}}_{r}\right) \leq$ $\lambda_{\text {max }}\left(\boldsymbol{k}_{2}\right)\left\|\boldsymbol{J}\left(\dot{\boldsymbol{q}}-\dot{\boldsymbol{q}}_{r}\right)\right\|^{2}$

where $\lambda_{\min }\left(\boldsymbol{k}_{2}\right)$ and $\lambda_{\max }\left(\boldsymbol{k}_{2}\right)$ are the minimum and the maximum eigenvalues of $\boldsymbol{k}_{2}$, respectively.

Using the Cauchy-Schwartz inequality and considering the upper bound of modelling error as $\left\|\boldsymbol{\varepsilon}_{f}\right\|<$ $\rho$, the left-hand side of (67) can be obtained

$-\left(\boldsymbol{J}\left(\dot{\boldsymbol{q}}-\dot{\boldsymbol{q}}_{r}\right)\right)^{T} \boldsymbol{\varepsilon}_{f}+\delta e^{-\sigma t} \leq\left\|\boldsymbol{J}\left(\dot{\boldsymbol{q}}-\dot{\boldsymbol{q}}_{r}\right)\right\|\left\|\boldsymbol{\varepsilon}_{f}\right\|+\delta<$

$\rho\left\|\boldsymbol{J}\left(\dot{\boldsymbol{q}}-\dot{\boldsymbol{q}}_{r}\right)\right\|+\delta$
Therefore, by considering (68) and (69) in order to satisfy $\dot{V}<0$

$\dot{V}<-\lambda_{\min }\left(\boldsymbol{k}_{2}\right)\left\|\boldsymbol{J}\left(\dot{\boldsymbol{q}}-\dot{\boldsymbol{q}}_{r}\right)\right\|^{2}+\delta+\rho\left\|\boldsymbol{J}\left(\dot{\boldsymbol{q}}-\dot{\boldsymbol{q}}_{r}\right)\right\|(70$

In other words

$$
\lambda_{\min }\left(\boldsymbol{k}_{2}\right)>\frac{\delta}{\left\|\boldsymbol{J}\left(\dot{\boldsymbol{q}}-\dot{\boldsymbol{q}}_{r}\right)\right\|^{2}}+\frac{\rho}{\left\|\boldsymbol{J}\left(\dot{\boldsymbol{q}}-\dot{\boldsymbol{q}}_{r}\right)\right\|}
$$

Let us define two diagonal positive definite matrices $\boldsymbol{k}_{21}$ and $\boldsymbol{k}_{22}$ such that $\boldsymbol{k}_{2}=\boldsymbol{k}_{21}+\boldsymbol{k}_{22}$ (i.e. $\lambda_{\min }\left(\boldsymbol{k}_{2}\right)=$ $\left.\lambda_{\text {min }}\left(\boldsymbol{k}_{21}\right)+\lambda_{\text {min }}\left(\boldsymbol{k}_{22}\right)\right)$, in order to satisfy

$$
\begin{aligned}
& \lambda_{\text {min }}\left(\boldsymbol{k}_{21}\right)>\frac{\delta}{\left\|J\left(\dot{\boldsymbol{q}}-\dot{\boldsymbol{q}}_{r}\right)\right\|^{2}} \\
& \lambda_{\text {min }}\left(\boldsymbol{k}_{22}\right)>\frac{\rho}{\left\|\boldsymbol{J}\left(\dot{\boldsymbol{q}}-\dot{\boldsymbol{q}}_{r}\right)\right\|}
\end{aligned}
$$

As a result, in order to satisfy (67), it is sufficient that

$$
\begin{gathered}
\sqrt{\frac{\delta}{\lambda_{\min }\left(\boldsymbol{k}_{21}\right)}} \triangleq \beta_{1}<\left\|\boldsymbol{J}\left(\dot{\boldsymbol{q}}-\dot{\boldsymbol{q}}_{r}\right)\right\| \\
\frac{\rho}{\lambda_{\min }\left(\boldsymbol{k}_{22}\right)} \triangleq \beta_{2}<\left\|\boldsymbol{J}\left(\dot{\boldsymbol{q}}-\dot{\boldsymbol{q}}_{r}\right)\right\|
\end{gathered}
$$

where $\beta_{1}$ and $\beta_{2}$ are positive constants. It is noted that $\dot{V}<0$ as long as $\beta<\left\|\boldsymbol{J}\left(\dot{\boldsymbol{q}}-\dot{\boldsymbol{q}}_{r}\right)\right\|$ in which $\beta=\max \left(\beta_{1}, \beta_{2}\right)$. This implies that the reference error $\boldsymbol{e}$ and its time derivative $\dot{e}$ become smaller out of the ball with the radius of $\beta$. As a result, the reference error and its time derivative are bounded and ultimately enters into the ball with the radius of $\beta$.

Considering assumption 2 and 3 and the proof given by [31], $\boldsymbol{v}, \boldsymbol{I}_{a}, \dot{\boldsymbol{I}}_{a}$ and $\dot{\boldsymbol{q}}$ are bounded. Since the desired impedance matrices $\boldsymbol{M}_{R}, \boldsymbol{B}_{R}$ and $\boldsymbol{K}_{R}$ are chosen in such a way as the roots of $\boldsymbol{M}_{R} s^{2}+\boldsymbol{B}_{R} s+\boldsymbol{K}_{R}$ be in the lefthand side of the s-plane and thus according to assumption 1, equations (56) and (26), $\boldsymbol{x}_{m}$ and $\dot{\boldsymbol{x}}_{m}$ are bounded. Since $\boldsymbol{e}, \dot{\boldsymbol{e}}, \boldsymbol{x}_{m}$ and $\dot{\boldsymbol{x}}_{m}$ are bounded, the boundedness of $\boldsymbol{x}$, $\dot{\boldsymbol{x}}, \boldsymbol{x}_{r}, \dot{\boldsymbol{x}}_{r}$ and $\ddot{\boldsymbol{x}}_{r}$ is proven. As a result, the regression matrix $\boldsymbol{Y}_{x}\left(\boldsymbol{x}, \dot{\boldsymbol{x}}, \dot{\boldsymbol{x}}_{r}, \ddot{\boldsymbol{x}}_{r}\right)$ and $\boldsymbol{Y}\left(\boldsymbol{q}, \dot{\boldsymbol{q}}, \dot{\boldsymbol{q}}_{r}, \ddot{\boldsymbol{q}}_{r}\right)$ are bounded and following that $\widehat{\boldsymbol{P}}$ is bounded by considering (63). The boundedness of equation (19), $\boldsymbol{e}$ and $\dot{\boldsymbol{e}}$ result in the boundedness of $\widehat{\psi}$ and $\hat{\eta}$ in equation (64) and (65), respectively. Thus, from (18) $\hat{\boldsymbol{\mu}}$ is bounded.

\section{Simulation results}

The proposed control approach is applied for the robust control of the electrically driven two-link elbow manipulator. The dynamic parameters of the robot and the motors' parameters have been presented in Table. 1 and Table 2, respectively. The desired position trajectory for end-effector, as illustrated in Fig. 2, is formulated by 


$$
\boldsymbol{x}_{d}=\left[x_{d 1}, x_{d 2}\right]^{T}
$$

where $x_{d 1}$ and $x_{d 2}$ are given as

$$
\begin{aligned}
& x_{d 1}=\left\{\begin{array}{cc}
0.65-0.65 \cos (\pi t / 3) & 0 \leq t<3 \\
1.3 & t \geq 3
\end{array}\right. \\
& x_{d 2}=1.3
\end{aligned}
$$
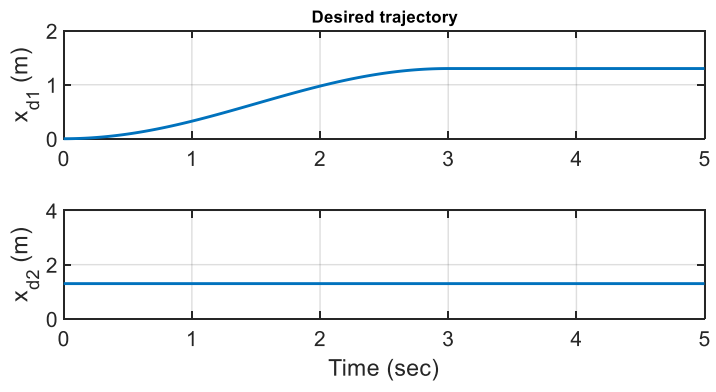

Fig. 2. Desired Trajectory

Remark 5. To perform simulation and according to Remark 4, one can use inverse kinematics in order to obtain the joint variables in terms of the $x$ and $y$ coordinates. Considering Fig. 3 and using the Law of cosines, we have

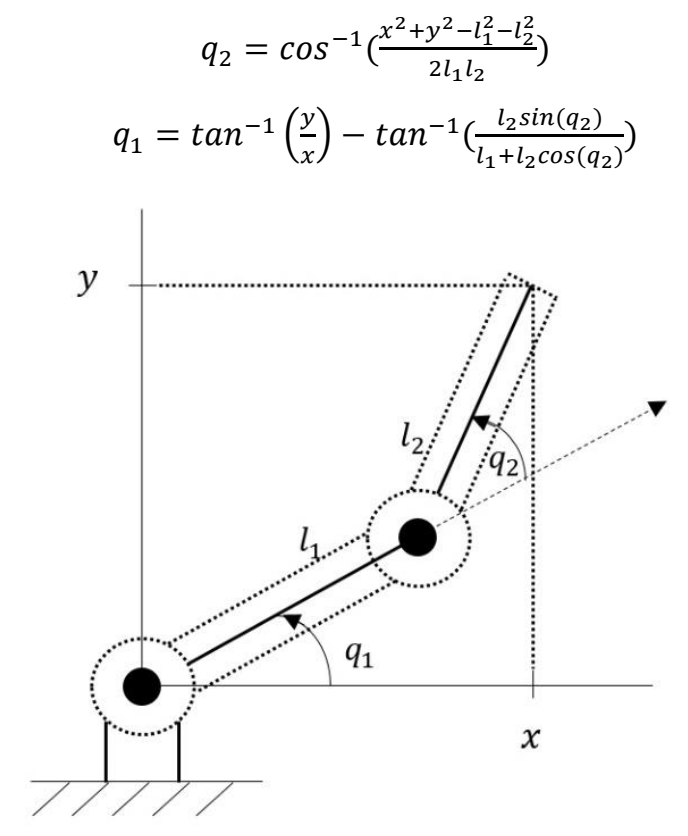

Fig. 3. A two-link planar arm
Table 1. Dynamic parameters of two-link planar arm

\begin{tabular}{|c|c|}
\hline Parameters & Value \\
\hline$l_{1}, l_{2}(\mathrm{~m})$ & 1 \\
\hline$l_{c 1}, l_{c 2}(\mathrm{~m})$ & 0.5 \\
\hline$m_{1}(\mathrm{~kg})$ & 15 \\
\hline$m_{2}(\mathrm{~kg})$ & 6 \\
\hline$I_{1}\left(\mathrm{~kg} \cdot \mathrm{m}^{2}\right)$ & 5 \\
\hline$I_{2}\left(\mathrm{~kg} \cdot \mathrm{m}^{2}\right)$ & 2 \\
\hline
\end{tabular}

Table 2. The specifications of permanent magnet dc motors.

\begin{tabular}{|c|c|}
\hline Parameters & Value \\
\hline$u_{\max }(\mathrm{V})$ & 40 \\
\hline$R(\Omega)$ & 1.6 \\
\hline$L(\mathrm{H})$ & 0.001 \\
\hline$K_{b}(\mathrm{~V} . \mathrm{s} / \mathrm{rad})$ & 0.26 \\
\hline$K_{m}(\mathrm{~N} . \mathrm{m} / \mathrm{A})$ & 0.26 \\
\hline$J_{m}\left(\mathrm{Nm} . \mathrm{s}^{2} / \mathrm{rad}\right)$ & 0.0002 \\
\hline$B_{m}(\mathrm{Nm} . \mathrm{s} / \mathrm{rad})$ & 0.001 \\
\hline$r$ & 0.02 \\
\hline
\end{tabular}

Simulation 1. The environment model with $\boldsymbol{x}_{\boldsymbol{e}}=\left[\begin{array}{ll}1.29 & \mathrm{y}\end{array}\right]^{T} m$ and $\boldsymbol{K}_{\boldsymbol{e}}=\operatorname{diag}(100000,0) \mathrm{N} / \mathrm{m}$ is given by

$$
\boldsymbol{K}_{e}\left(\boldsymbol{x}-\boldsymbol{x}_{e}\right)=\boldsymbol{F}_{e}
$$

where $\boldsymbol{x}_{e} \in R^{m}$ is the equilibrium position, and $\boldsymbol{K}_{e}$ is a $n \times n$ diagonal matrix which is called the stiffness of the environment. The model reference parameters in (25) are set to $\boldsymbol{M}_{R}=5.4 I_{2} \mathrm{Ns}^{2} / \mathrm{m}, \boldsymbol{B}_{R}=1500 I_{2} \mathrm{Ns} / \mathrm{m}$, and $\boldsymbol{K}_{R}=500 I_{2} \mathrm{~N} / \mathrm{m}$. The control approach parameters are set to $\boldsymbol{k}_{1}=\operatorname{diag}(125,125), \boldsymbol{k}_{2}=\operatorname{diag}(2.8,4), \boldsymbol{\Gamma}=$ $\operatorname{diag}\left(0.01 I_{5}, 0.1\right), \gamma_{\eta}=0.1, \sigma=0.2, \delta=0.1$ by trial and error and the time delay is set to $\varepsilon=0.001 \mathrm{sec}$. The initial values for vector $\widehat{\boldsymbol{P}}(0)$ are selected as $\widehat{\boldsymbol{P}}(0)=$ 1.1P. Furthermore, the parameters of fuzzy system are chosen as $\gamma_{f}=0.8$ and $\sigma_{f}=0.55$. The external disturbance profile $\boldsymbol{\varphi}(t)$ is defined as a step function and is applied in $t=1.5 \mathrm{sec}$. The selected amplitude for external disturbance is chosen based on approximately $10 \%$ of the maximum voltage amplitude. The initial position for the end-effector is set to $\left(x_{0}, y_{0}\right)=$ $(-0.059 \mathrm{~m}, 1.259 \mathrm{~m})$. The Schematic plot of robot manipulator trajectory is shown in Fig. 4. As shown in 
Fig. 4, the proposed controller should compensate the initial position of the end-effector to put it into the straight-line trajectory and finally the end-effector will interact the environment.

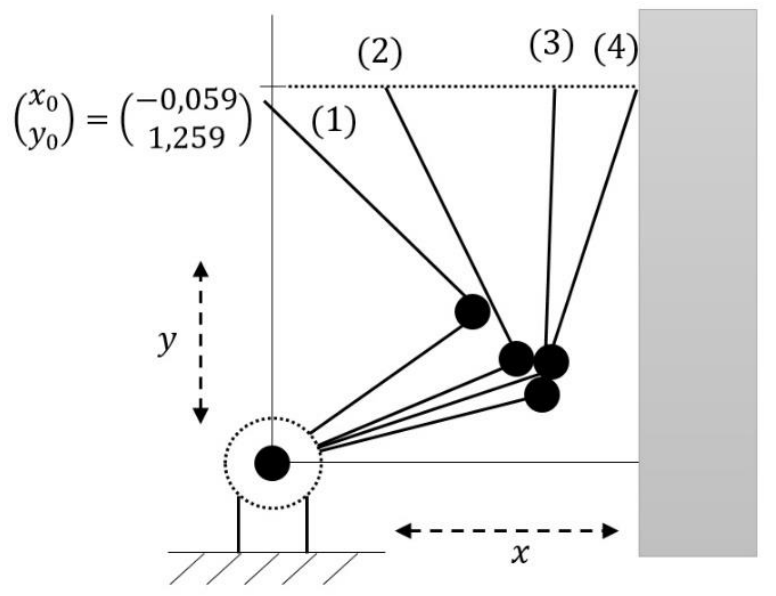

Fig. 4. Schematic plot of robotic trajectory

The contact force between the end-effector and environment is depicted in Fig .5. As shown, the contact phenomenon occurs in $2.84 \mathrm{sec}$ and the generated force reaches rapidly a maximum value of $122.1 \mathrm{~N}$. From this time forward, the proposed compensator comes into operation and efficiently compensates the harmful effect of the contact and finally makes the contact force approximately decrease to $4 \mathrm{~N}$.

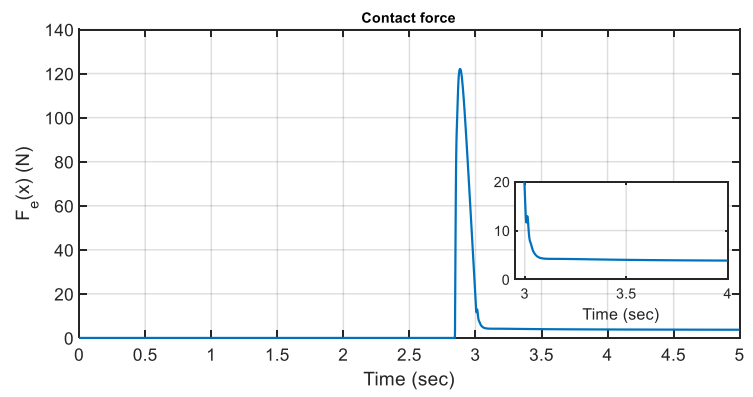

Fig. 5. The contact force between the end-effector and environment

Motors behave well under the permitted voltages as shown in Fig. 6. The interaction effect of end-effector and environment for both motors' voltages at $t=2.84 \mathrm{sec}$ is detectable. Also, it is noted that from the contact between the end-effector and environment forward, both motors approximately tolerate constant voltages. Fig. 7 illustrates the performance of control approach providing similar behavior of robotic system and the reference model such that the reference errors are reduced well to the range of $\left[\begin{array}{ll}-2.5 & 2.5\end{array}\right] \times 10^{-3} \mathrm{~m}$. The tracking performance of the reference model is shown in Fig 8. The first reference model tracking error, i.e. $\boldsymbol{x}_{d 1}-\boldsymbol{x}_{m 1}$, shows that the contact between the end effector and the environment occurs at $t=2.84 \mathrm{sec}$ along the $x$ axis. The second signal of reference model, $\boldsymbol{x}_{m 2}$, does not show any deviation from the desired signal, $\boldsymbol{x}_{d 2}$, since there is no force along the $y$ axis. It is noted that by increasing the first model reference tracking error, the control approach protects the robot and the environment from the possible detrimental effect of the contact. To do this, according to Figure (5) and Figure (8), the contact force finally decreases to $4 N$ such that the second signal of reference model for the end-effector is deviated approximately $9 \mathrm{~mm}$ along the $x$ axis. Figure. 9 shows the adaptive fuzzy parameters (Eq. 64), $\widehat{\boldsymbol{\psi}}=\left[\widehat{\boldsymbol{\psi}}_{1}, \ldots, \widehat{\boldsymbol{\psi}}_{27}\right]^{T}$, which all parameters finally converge to constant values. The adaptation of $\hat{\eta}$ (Eq. 65) and $\widehat{\boldsymbol{P}}$ (Eq. 63) is also depicted in Fig. 10 and Fig. 11, respectively. The effectiveness of the proposed controller is shown by simulation results.

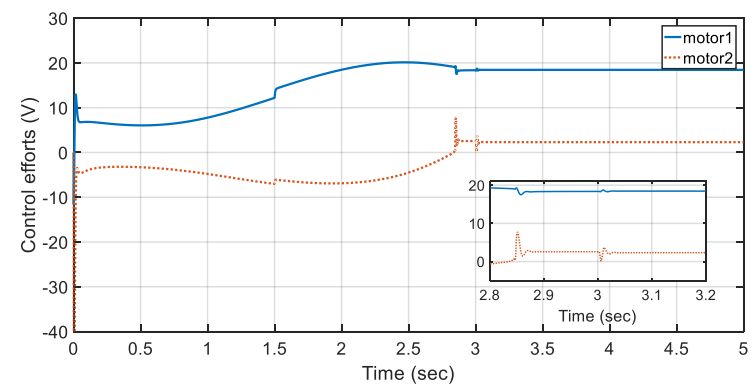

Fig. 6. Control efforts of the proposed control approach

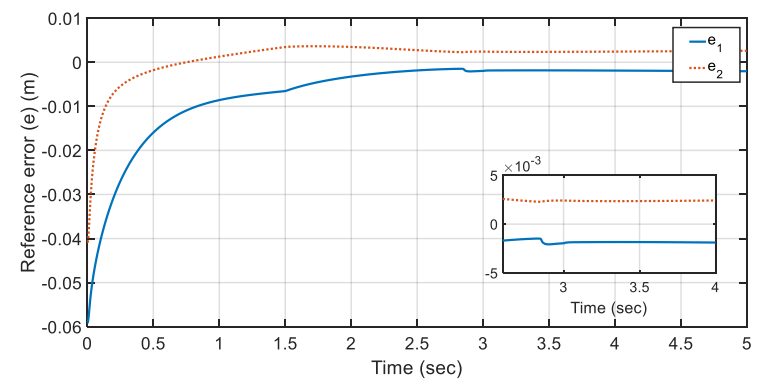

Fig. 7. The reference errors (Eq. 34) 

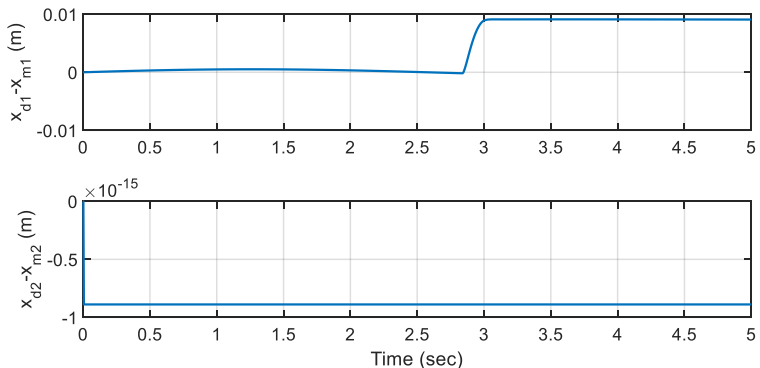

Fig. 8. Tracking performance of the reference model

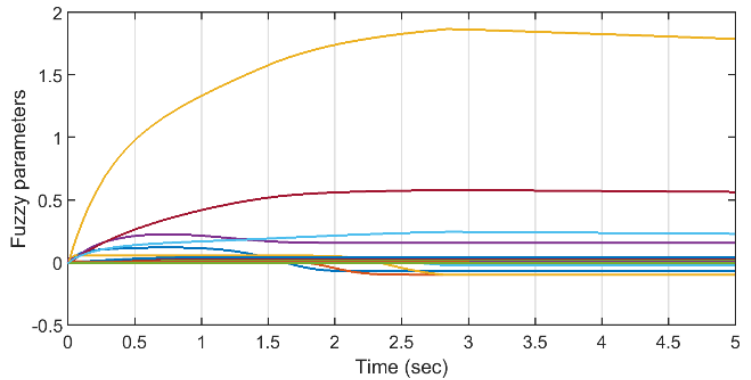

Fig. 9. Adaptation of uncertainty fuzzy parameters

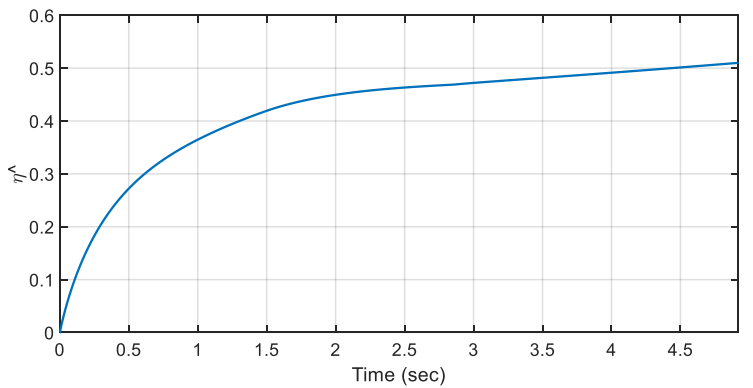

Fig. 10. Adaptation of parameter $\hat{\eta}$

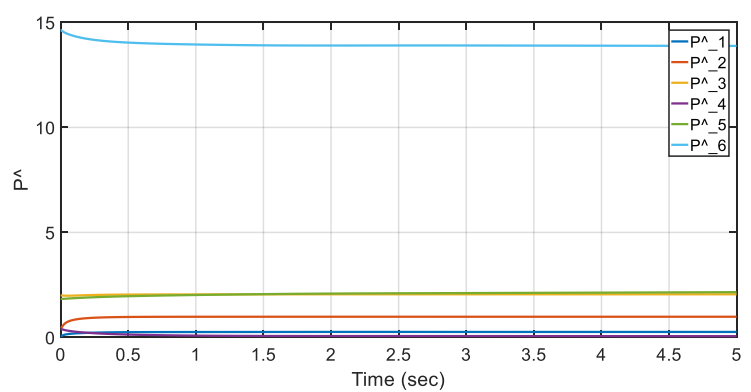

Fig. 11. Adaptation of $\widehat{\boldsymbol{P}}$

Simulation 2. The performance of the proposed controller is evaluated by different stiffness of the environments. To perform simulation, four values for $\boldsymbol{K}_{\boldsymbol{e}}$ are chosen as $\boldsymbol{K}_{e 1}=\operatorname{diag}(20000,0), \quad \boldsymbol{K}_{e 2}=$ $\operatorname{diag}(60000,0), \boldsymbol{K}_{e 3}=\operatorname{diag}(100000,0)$ and $\boldsymbol{K}_{e 4}=$ $\operatorname{diag}(140000,0)$. Fig. 12 and Fig. 13 illustrate the contact force between the end-effector and different environments and the performance of the control method interacting with environments with different stiffness, respectively.

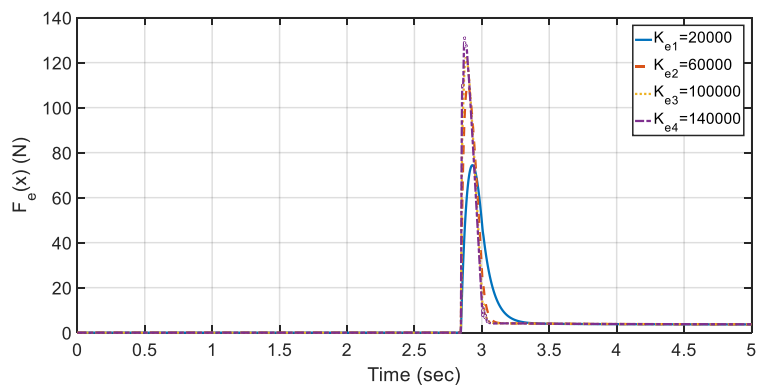

Fig. 12. The contact force between the end-effector and environments with different stiffness

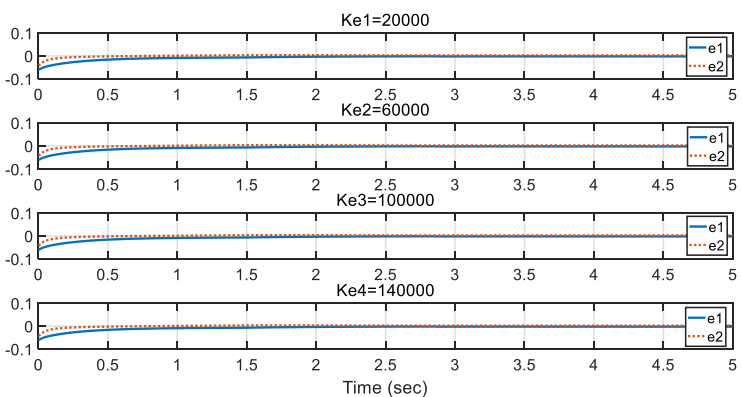

Fig. 13. The performance of the control method interacting with environments with different stiffness

\section{Conclusion}

This paper has proposed a model-reference impedance control approach using fuzzy uncertainty estimator for the robust control of electrically driven robot manipulators. In the proposed control method, a desired dynamical behavior of the robot interacting with an environment is considered as the model-reference such that this model-reference provides a reference signal for a robot whether in tracking or contact situations. As the interaction between an end-effector and an environment occurs, the contact force is exerted to the modelreference and the robotic system receives the output of model-reference as a desired trajectory and thus the aim of the controller is to reduce the difference between the task space desired trajectory and the desired impedance model. It is worth noting that the feedback of contact force is not utilized in the structure of the proposed controller and the adaptive laws since by increasing the contact force at the time of the interaction between an end-effector and an environment, existing the contact 
force will cause problematic issues for control designers. Furthermore, to improve the performance of control system and also compensate un-modelled dynamics and external disturbances, two control terms namely a robustifying term and a fuzzy uncertainty estimator are employed in the structure of the controller. The proposed control approach has shown a good performance in terms of simplicity in design, high-performance of contact force compensation and small reference error. The stability analysis has been guaranteed by the proposed control method and the simulation results verified the effectiveness of the method.

\section{Appendix A.}

The regressor matrix $\boldsymbol{Y}\left(\boldsymbol{q}, \dot{\boldsymbol{q}}, \dot{\boldsymbol{q}}_{r}, \ddot{\boldsymbol{q}}_{r}\right)$ is formulated as

$\boldsymbol{Y}\left(\boldsymbol{q}, \dot{\boldsymbol{q}}, \dot{\boldsymbol{q}}_{r}, \ddot{\boldsymbol{q}}_{r}\right)=\left[\begin{array}{llllll}Y_{11} & Y_{12} & Y_{13} & Y_{14} & Y_{15} & Y_{16} \\ Y_{21} & Y_{22} & Y_{23} & Y_{24} & Y_{25} & Y_{26}\end{array}\right]$ (A.1)

where

$$
\begin{gathered}
Y_{11}=Y_{13}=\ddot{q}_{r 1} \\
Y_{12}=Y_{22}=\ddot{q}_{r 1}+\ddot{q}_{r 2} \\
Y_{14}=2 l_{1} \cos \left(q_{2}\right) \ddot{q}_{r 1}+l_{1} \cos \left(q_{2}\right) \ddot{q}_{r 2}- \\
l_{1} \sin \left(q_{2}\right) \dot{q}_{2} \dot{q}_{r 1}-l_{1} \sin \left(q_{2}\right)\left(\dot{q}_{1}+\dot{q}_{2}\right) \dot{q}_{r 2}+ \\
g \cos \left(q_{1}+q_{2}\right) \\
Y_{15}=g \cos \left(q_{1}\right), Y_{16}=\dot{q}_{r 1}, Y_{21}=\ddot{q}_{r 2} \\
Y_{23}=Y_{25}=0 \\
Y_{24}=l_{1} \cos \left(q_{2}\right) \ddot{q}_{r 1}+l_{1} \sin \left(q_{2}\right) \dot{q}_{1} \dot{q}_{r 1} \\
+g \cos \left(q_{1}+q_{2}\right) \\
Y_{26}=\dot{q}_{r 2}
\end{gathered}
$$

\section{References:}

1. M. H. Raibert and J. J. Craig, Hybrid position/force control of manipulators, J. Dyn. Syst. Meas. Contr. 103(2) (1981) 126-133.

2. J. K. Mills and A. A. Goldenberg, Force and position control of manipulators during constrained motion tasks, IEEE Trans. Rob. Autom. 5(1) (1989) 30-46.

3. H. Wang and Y. Xie, Adaptive Jacobian force/position tracking control of robotic manipulators in compliant contact with an uncertain surface, $A d v$. Rob. 23(1-2) (2009) 165-183.

4. N. Hogan, Impedance control: An approach to manipulation: Part II-Implementation, J. Dyn. Syst. Meas. Contr. 107(1) (1985) 8-16.
5. W. S. Lu and Q. H. Meng, Impedance control with adaptation for robotic manipulations, IEEE Trans. Rob. Autom. 7(3) (1991) 408-415.

6. R. Colbaugh, H. Seraji and K. Glass, Direct adaptive impedance control of robot manipulators, J. Rob. Syst. 10 (2) (1993) 217-248.

7. L. Sciavicco and B. Siciliano. Modelling and control of robot manipulators. (Springer Science \& Business Media. 2012)

8. S. M. Ahmadi and M. M. Fateh, Robust control of electrically driven robots using adaptive uncertainty estimation, Comput. Electr. Eng. 56 (2016). 674-687.

9. M. C. Chien and A. C. Huang, Adaptive impedance control of robot manipulators based on function approximation technique, Robotica, 22(04) (2004) 395403.

10. A. Ibeas and M. De la Sen, Robust impedance control of robotic manipulators. 43rd IEEE Conf. In Decision and Control (2004), pp. 1258-1263.

11. C. C. Cheah and D. Wang, Learning impedance control for robotic manipulators. IEEE Trans. Rob. Autom. 14(3) (1998) 452-465.

12. S. Jung, T. C. Hsia and R. G. Bonitz, Force tracking impedance control of robot manipulators under unknown environment, IEEE Trans. Contr. Syst. Technol. 12(3) (2004) 474-483.

13. C. Y. Su, T. P. Leung and Y. Stepanenko, Real-time implementation of regressor-based sliding mode control algorithm for robotic manipulators. IEEE Trans. Ind. Electron. 40(1) (1993) 71-79.

14. B. S. Chen, H. J. Uang and C. S. Tseng, Robust tracking enhancement of robot systems including motor dynamics: A fuzzy-based dynamic game approach, IEEE Trans. Fuzzy Syst. 6(4) (1998) 538-552.

15. S. P. Chan and H. P. Chen, Robust implementation of generalized impedance control for robot manipulators, Adv. Rob. 15(6) (2001) 641-661.

16. M. M. Fateh, On the voltage-based control of robot manipulators, Int. J. Cont. Autom. Syst. 6(5) (2008) 702 712.

17. M. M. Fateh and M. Souzanchikashani, Indirect adaptive fuzzy control for flexible-joint robot manipulators using voltage control strategy, J. Intell. Fuzzy Syst. 28(3) (2015) 1451-1459.

18. M. M. Fateh, and R. Babaghasabha, Impedance control of robots using voltage control strategy, Nonlinear Dyn. 74(1-2) (2013) 277-286.

19. Z. Li, J. Liu, Z. Huang, Y. Peng, H. Pu, and L. Ding, Adaptive Impedance Control of Human-Robot Cooperation Using Reinforcement Learning, IEEE Trans. Ind. Electron. (2017).

20. W. He, Y. Dong and C. Sun, Adaptive neural impedance control of a robotic manipulator with input saturation, IEEE Trans. Syst. Man Cybern. Syst. 46(3) (2016) 334 344. 
21. H. R. Koofigar, Adaptive tracking with external force disturbance rejection for uncertain robotic systems, Int. J. Cont. Autom. Syst. 12(1) (2014) 169.

22. Z. Y. Jhan, C. H. Lee and C. M. Lin, A new adaptive fuzzy neural force controller for robots manipulator interacting with environments, IEEE Int. Conf. In Machine Learning and Cybernetics (2015), pp. 572-577.

23. M. H. Ghajar, M. Keshmiri and J. Bahrami, Neuralnetwork-based robust hybrid force/position controller for a constrained robot manipulator with uncertainties, Trans. Inst. Meas. Control (2017).

24. A. Fanaei and M. Farrokhi, Robust adaptive neuro-fuzzy controller for hybrid position/force control of robot manipulators in contact with unknown environment, $J$. Intell. Fuzzy Syst. 17(2) (2006) 125-144.

25. Z. Hu, R. Bicker and C. Marshall, Position/force control of manipulator based on force measurement and its application to gear deburring, J. Intell. Fuzzy Syst.14(4) (2003) 215-223.

26. M.W. Spong, S. Hutchinson and M. Vidyasagar, Robot modeling and control (New York: Wiley, 2006).

27. L. X. Wang, A course in fuzzy systems, in Prentice-Hall press (USA, 1999), pp. 258-265.

28. Z. Zheng, S. Wu, W. Liu and K. Y. Cai, A feedback based CRI approach to fuzzy reasoning. Appl. Soft Comput. 11(1) (2011) 1241-1255.

29. S. M. Ahmadi and M. M. Fateh, Task-space control of robots using an adaptive Taylor series uncertainty estimator, Int. J. Control (2018) https://doi.org/10.1080/00207179.2018.1429673.

30. M. M. Fateh, S. M. Ahmadi and S. Khorashadizadeh, Adaptive RBF network control for robot manipulators, $J$. AI. Data Min. 2(2) (2014) 159-166.

31. M. M. Fateh, Robust control of flexible-joint robots using voltage control strategy, Nonlinear Dyn. 67(2) (2012) 15251537. 